\title{
Molecular Dynamics Investigation of Structure Evolution and Thermodynamics of Ni-fe Nanoparticles During Inert Gas Condensation
}

Bei Li ( $\sim$ libei@whut.edu.cn )

Wuhan University of Technology https://orcid.org/0000-0002-8242-9657

Lei Pan

Wuhan University of Technology

Changan Liu

Wuhan University of Technology

Xu Zhang

Wuhan University of Technology

\section{Research Article}

Keywords: nucleation, cluster growth, aggregation, solidification

Posted Date: March 30th, 2021

DOI: https://doi.org/10.21203/rs.3.rs-335602/v1

License: (c) (i) This work is licensed under a Creative Commons Attribution 4.0 International License. Read Full License 


\section{Abstract}

Synthesis of magnetic nanoparticles is relevant to many applications in the fields of catalysis, energy storage and biomedicine, etc. Understanding the growth mechanisms and morphology of nanoparticles during inert gas condensation is crucial to rationally improve the performance of the final nanoparticles. In this work, molecular dynamics simulations are carried out to study the structural and thermodynamic behavior of Ni-Fe nanoparticles from homogenous vapor phase in Ar atmosphere. It is revealed that the final morphology of the resulting nanoparticles presents a spherical shape by cluster coalescence at high temperatures where the small clusters are liquid droplets prior to their collisions. However, probabilistic nucleation and cluster growth indicate that the occurrence of spherical shape is more controlled by the probability limits for different Fe concentrations. Meanwhile, a larger inert gas density induces a more efficient cooling effect leading to a larger probability control of the cluster formation with non-spherical shape by agglomeration. Furthermore, the solidification of the as-formed Ni-Fe clusters is examined by evaluating the evolution of crystalline and amorphous structure. The linear scaling-down dependence of the solidification temperature on the reciprocal of the nanoparticle size clearly signifies a linear sizedepression effect for the liquid-to-solid phase change of Ni-Fe nanoparticles. Our findings thus extend the current understanding of inert gas condensation behavior and mechanisms of Ni-Fe nanoparticles from an atomic/molecular perspective.

\section{Introduction}

In recent decades, metal nanoparticles have been the subject of scientific and technical interest because of their attractive physical and chemical properties as compared to the macroscopic counterparts due to large surface area to volume ratios and active surface atoms. Among them, magnetic nanoparticles have drawn considerable attention in numerous areas such as high energy products [1], solar cells [2, 3], spin electronics [4-6], catalysis [7-9], bio-sensing and biomedicine [10-12], etc. Since all these applications rely on their size and internal structure, knowledge of preparation and characterization of the magnetic nanoparticles with controllable size, shape, composition and crystalline structure is significantly crucial from the viewpoint of both fundamental research and industrial applications.

Many methods have been proposed and explored to produce well-characterized magnetic nanoparticles such as hydrothermal reaction, electrodeposition, laser ablation and inert gas condensation, etc. [13-19]. In particular, the synthesis of nanoparticles from the vapor phase in inert gas is regarded as one of the most versatile and flexible techniques by tuning growth process conditions such as magnetron power, cluster-beam drift velocity, aggregation length and carrier gas density [20, 21]. The metals evaporation and condensation in cooling inert gas atmosphere could fabricate nanoparticles with a certain size, shape, composition and structure in an economic and environmentally friendly way. For example, Singh et al. reported experimental work on Si-Ag nanoparticles and found that the size and quantity of $\mathrm{Ag}$ domains were manipulated by varying the Ag magnetron sputtering power, resulting in Janus and coresatellite structures [22]. Bohra et al. described the gas phase synthesis of $\mathrm{Ni}-\mathrm{Cr}$ nanoparticles and the potential to tune Curie temperature within a wide temperature range by modifying the $\mathrm{Cr}$ concentrations 
[23]. It showed preferential full surface segregation of $\mathrm{Cr}$ up to 5 at. \% $\mathrm{Cr}$, but unexpectedly, the presence of residual $\mathrm{Cr}$ inside the Ni-rich cores for higher concentrations. Golkar et al. synthesized sub-10-nm Co-W clusters using inert gas condensation and studied the effects of sputtering power and temperature on the average sizes and structure of the clusters as well as their corresponding magnetic properties [24]. Furthermore, the thermodynamics and mechanisms of metallic nanoparticle preparation using inert gas condensation have been investigated using computational techniques. Lümmen et al. studied the inert gas condensation behaviors of Fe nanoparticles using molecular dynamics (MD) method and observed an increase in the amount of carrier gas could accelerate the cluster growth [25]. Kesälä et al. evaluated the growth speed of $\mathrm{Cu}, \mathrm{Ag}$ and $\mathrm{Al}$ clusters during inert gas condensation using MD simulations and reported an inversely proportional relation between the condensation time and the initial partial vapor pressure [26]. Abbaspour et al. performed a series of MD simulations of Ni-Pt, Pd-Pt and Au-Ir-Pd nanoparticles and suggested that the condensation conditions, i.e., the metal mole fraction, temperature and pressure, play an important role in the manufacturing process as well as the resulting structural and thermodynamic properties [27-29].

In spite of these experimental and computational investigations on the inert gas condensation procedure, little has been done to gain a comprehensive understanding on the inert gas condensation of magnetic nanoparticles, in particular in the structural behaviors and growth mechanisms. Here, the Ni-Fe alloy model is one of the most common soft magnetic materials, showing excellent magnetic properties, i.e., high saturation magnetization, high permeability and low energy loss [30], and has been widely applied in the fields of electronic, medical, nuclear, chemical and petrochemical industries [31,32]. Therefore, in this work, we employ MD simulations to investigate the inert gas condensation of Ni-Fe nanoparticles from metastable vapor phase. The effects of the compositions of metal atoms and the amount of carrier gas on the nucleation, cluster growth and phase transition of Ni-Fe systems in the Ar atmosphere will be systematically investigated from an atomic/molecular perspective.

\section{Simulation Details}

\subsection{Molecular models}

The initial configurations for simulation of formation of Ni-Fe magnetic nanoparticles were constructed by placing $\mathrm{Fe}, \mathrm{Ni}$ and $\mathrm{Ar}$ atoms stochastically in a cubic cell with $35 \mathrm{~nm}$ lengths in the three dimensions. The total number of the metal atoms in all the simulations was 1500 , giving rise to an initial metal vapor density of $0.058 \mathrm{~mol} / \mathrm{dm}^{3}$. In order to simulate different initial compositions of the system, six different concentrations of Fe atoms were chosen as 5, 10, 15, 20, 25 and $30 \mathrm{wt}$ \% and the ratio of the number of Ar to metal atoms (Ar/Metal ratio) was maintained at 1:1 to mimic the inert gas atmosphere. Especially for the system with $30 \mathrm{wt} . \% \mathrm{Fe}$, we also introduced 1125, 1500, 3000, 4500 and $6000 \mathrm{Ar}$ atoms in the simulation box, corresponding to varying inert gas densities in a range of $0.044-0.232 \mathrm{~mol} / \mathrm{dm}^{3}$. Figure 1 illustrates a typical equilibrated inert-gas-metal-vapor mixture consisting of $1500 \mathrm{Ar}$ atoms and 1500 metal atoms with $30 \mathrm{wt} . \%$ Fe at $T=4000 \mathrm{~K}$. 


\subsection{Simulation method}

To describe the interactions between metal atoms, we used the embedded atom method (EAM) potential as introduced by Bonny et al. [33]. This EAM-method has been successfully employed for simulation of static and dynamic properties for systems containing $\mathrm{Fe}, \mathrm{Ni}$ and $\mathrm{Cr}$ atoms, such as lattice parameters, diffusion coefficient, thermal conductivity, surface melting and $\mathrm{Cr}$ segregation, etc. [34-38]. Moreover, the 12 - 6 Lennard-Jones potential was used to compute the van der Waals interactions between Ar atoms, whereas the interactions between metal and Ar atoms were represented by a universal, purely repulsive potential developed by Ziegler, Biersack and Littmark (ZBL) [39].

Initially, the random distributed inert-gas-metal-vapor system was equilibrated at $T=4000 \mathrm{~K}$ for $100 \mathrm{~ns}$ using the Nose-Hoover thermostat with a time constant of $0.1 \mathrm{ps}$. The long simulation time was sufficient for metal atoms to reach a metastable vapor phase. After equilibration, the Ni-Fe nanoparticles were condensed in the inert gas atmosphere at $T=300 \mathrm{~K}$. To characterize the condensation realistically, only the Ar atoms were treated as the heat bath and were coupled to the Berendsen thermostat to control the temperature. The metal atoms were cooled down by elastic collisions with colder Ar atoms without any other thermostat applied. The total simulation time for the inert gas condensation process was $200 \mathrm{~ns}$ to fully relax the resulting Ni-Fe nanoparticles. The Newton's equations of motion were integrated using the Verlet leapfrog algorithm with a time-step of $1.0 \mathrm{fs}$ and periodic boundary conditions were used in all three dimensions.

\section{Results And Discussions}

\subsection{Nucleation and surface growth}

It is known that the metal nanoparticle synthesis during inert gas condensation usually consists of four characteristic stages, i.e., cluster nucleation, monomeric and surface growth, coalescence or aggregation. To demonstrate the cluster nucleation, the number of the clusters higher than or equal to a specific size is calculated within the first $2 \mathrm{~ns}$, as shown in Fig. 2 for $30 \mathrm{wt} . \%$ Fe and Ar/Metal $=1: 1$. For different threshold values $(5,7,9$ and 11 atoms), the cluster number increases quickly due to the negligible energy barrier and has an appropriately linear relation with the simulation time. Once all the nuclei are almost formed, growth ensues by adatom decoration and surface adsorption (see the insets in Fig. 2) leading to the gradual decrease in cluster quantities. Meanwhile, there exist fluctuations in the cluster number due to the simultaneous events of association and dissociation of the metal atoms at initial high temperatures. Here, the cluster nucleation rates were estimated as the slope of the initial linear fitting per unit volume and their values at different compositions and inert gas densities are presented in Figs. 3(a) and (b). As expected, the nucleation rate decreases with the threshold and achieves a steady value after a specific size. For different Fe concentrations, the nucleation steady rates are almost equivalent due to the similar interaction strengths for $\mathrm{Fe}$ and $\mathrm{Ni}$ atoms, which can be seen from the comparable radial distribution function (RDF) profiles of the metal atoms in Fig. S1. Contrarily, a larger amount of inert gas induces 
more energetic collisions between the metal and Ar atoms, thereby leading to a higher steady nucleation rate and stronger dependence on the threshold.

\subsection{Cluster aggregation and morphology}

In addition to the nucleation and surface growth in the very beginning, Ni-Fe clusters continue to evolve for around 180-190 ns, and the maximum cluster size versus simulation time $t$ is shown in Fig. 4 . The stochastic jumps in the size of the largest cluster correspond to the aggregation of the small clusters via coalescence (the structure of the clusters changes) or agglomeration (the structure and the shape remain without changes). More importantly, both the metal composition and inert gas density have a negligible influence on the statistical nature of the cluster aggregation, signifying a thermodynamic probabilitysieve growth mechanism that has been observed for bimetallic Ag-Au clusters from homogenous vapor phase [40]. To further illustrate the cluster aggregation, the formation snapshots of the Ni-Fe clusters at different metal temperatures $T$ are shown in Figs. 5 and S2. The metal atoms dissolve mutually both in the interior and at the surface as a result of the stronger $\mathrm{Ni}$-Fe interaction as compared to $\mathrm{Ni}-\mathrm{Ni}$ and $\mathrm{Fe}-\mathrm{Fe}$ ones (see Fig. S1). It is also worthy to note, the final morphology of the resulting Ni-Fe nanoparticles varies dramatically with different Fe concentrations and inert gas densities. After the completion of the condensation process, all the metal atoms assemble together and exhibit a spherical, dumbbell-like or wormlike shape with varying internal structure. For all the compositions, many primary clusters form at a wide range of $T=2000-2800 \mathrm{~K}$, and then eventually aggregate into larger spherical particles at high temperatures (1971 and $1361 \mathrm{~K}$ for compositions at 10 and $25 \mathrm{wt} . \% \mathrm{Fe}$ ) or non-spherical bizarre ones at low temperatures ( $<700 \mathrm{~K}$ for other concentrations). The formation of the Ni-Fe nanoparticles at different inert gas densities (Ar/Metal ratios) also suggest a rather high temperature is the key factor for the synthesis of spherical nanoparticles governed by coalescence. As seen from Fig. 5(a), the small clusters ( $N=99$ and $N=291$ ) collide to fuse together at $T=2241 \mathrm{~K}$, and the fused liquid particle quickly evolves into a unified spherical-like form at relatively high temperatures (i.e., $T>T_{\mathrm{S}}$, with $T_{\mathrm{S}}$ being the solidification temperature of the primary cluster) with high diffusivities. This corresponds to the growth mode of coalescence and also contributes to the aggregation of clusters of $N=767,390$ and 342 at $T=1971 \mathrm{~K}$, in which the initial clusters are all at liquid state and diffuse to mix and form a common particle with a different shape upon their collision. In this way, the metal vapor with $10 \mathrm{wt} . \%$ Fe finally produces a spherical-like crystalline nanoparticle at $T=506 \mathrm{~K}$. The same phenomenon is observed for $25 \mathrm{wt}$. Fe or low inert gas densities (see Fig. S2) as well. However, using a different initial configuration, the metal vapor phase with $10 \mathrm{wt} . \%$ Fe may result in a non-spherical or oval shape, while a spherical form is contrarily obtained at $5 \mathrm{wt}$.\% Fe (see Fig. S3), signifying the appearance of a spherical-like shape is a probability controlled process and is independent of the metal composition. On the other hand, a larger amount of inert gas induces a faster cooling effect on the metal atoms, thereby resulting in the formation of solid clusters prior to their collisions and subsequently anomalous shape by the way of agglomeration at $T<T_{\mathrm{s}}$ (i.e., the combination of the clusters of $N=678,353,183$ and 267 at $T<739 \mathrm{~K}$ in Fig. 5(d)).

\subsection{Size-dependent solidification}


The final morphology of the created Ni-Fe nanoparticles depends on the state (i.e., liquid or solid phase) of the small clusters prior to their collisions. It is therefore of significantly importance to investigate the solidification behavior of the clusters during the course of the simulation run. The evolution of the FCC, HCP and amorphous metal atoms as functions of the simulation time is presented in Fig. 6 . It is notable that the percentage of amorphous atoms drops abruptly at certain times, while the FCC and HCP atoms increase dramatically, indicating the occurrence of near-core preordering of the as-formed small clusters at elevated temperatures $T_{s}$. As seen in the CNA images (i.e., $i$ and ii in Figs. $6(b)$ and (d)), the clusters begin to crystalize at $T_{\mathrm{s}}$ in the inner region and then the transformation proceeds gradually to the surface. The solidification temperature of these specific clusters is thus defined as $T_{\mathrm{S}}$ (see Tables S1 and S2), to be consistent with previous studies [38, 41,42]. Also, there exists structural fluctuations in all the curves in Fig. 6 as the clusters collide or aggregate upon elastic collisions. However, the structural fluctuations become weakened at a larger inert gas density, ascribing to a more efficient cooling effect. In addition to the liquid-to-solid phase transition, the cluster aggregation is also able to release latent heat and raise up the metal temperature spontaneously until the residual heat is removed by the carrier gas. In this way, the abrupt changes in the crystalline and amorphous structure (iii and iv in Fig. 6(b)) are observed as a result of the heating and further thermal relaxation. After that, the crystalline structure remains almost unaltered leaving $36 \%-55 \%$ amorphous atoms on the surface, depending on the final morphology of the Ni-Fe nanoparticles.

Furthermore, the solidification temperature $T_{\mathrm{S}}$ of these specific clusters decreases with the cluster size $N_{\mathrm{S}}$. By plotting $T_{\mathrm{S}}$ versus $N_{\mathrm{S}}^{-1 / 3}$ of the Ni-Fe clusters, a linear relation is observed in Fig. 7, which agrees well with the MD simulation results for $\mathrm{Au}, \mathrm{Cu}, \mathrm{Ni}$ and Fe-Ni-Cr nanoparticles [38, 43, 44]. Although the discrepancy exists due to thermal fluctuation, $T_{\mathrm{s}}$ exhibits a rather strong dependence on the cluster size, but less so on the metal compositions and inert gas densities. More importantly, the linear scaling-down relation between $T_{\mathrm{s}}$ and $N_{\mathrm{s}}^{-1 / 3}$ indeed manifests the linear size-depression effect for the liquid-to-solid phase change of the Ni-Fe nanoparticles under different metal compositions and inert gas densities.

\section{Conclusion}

In this work, we have performed MD simulation of the inert gas condensation of the Ni-Fe nanoparticles from homogenous vapor phase in Ar atmosphere. The effects of different metal compositions and inert gas densities on the condensation behavior were investigated as well. The nucleation occurs rapidly and the steady nucleation rate was comparable for different compositions, while a larger Ar density induces a higher steady nucleation rate and stronger dependence on the threshold. For all the models, the metal vapor was condensed into one single cluster presenting spherical-like, dumbbell-like or wormlike shape with varying internal structure. Despite a high temperature is the key factor for the synthesis of spherical nanoparticles governed by coalescence, the occurrence of spherical morphology is rather a probability controlled process and is independent of the composition. Meanwhile, a larger inert gas density induces a faster cooling effect eliminating the probability of forming liquid clusters prior to their collisions and thus leading to the anomalous shape by the way of agglomeration due to insufficient thermal motion at low 
temperatures. Additionally, the solidification of the Ni-Fe clusters was examined by evaluating the crystalline structure evolution. A depression effect was observed for smaller clusters and the linear sizescaling down relation between the solidification temperature and the reciprocal of the nanoparticle size was verified. The findings in this work was believed to provide an atomic-scale investigation into the nucleation, cluster growth and phase transition of the Ni-Fe nanoparticles during inert gas condensation.

\section{Declarations}

Funding information This work is supported by the National Natural Science Foundation of China (No. 51603160) and the Open Fund Project of State Key Laboratory of Materials Processing and Die \& Mould Technology, Huazhong University of Science and Technology (No. P2021-009).

Conflicts of interest/Competing interests The authors declare that they have no known competing financial interests or personal relationships that could have appeared to influence the work reported in this paper.

Availability of data and material The raw/processed data required to reproduce these findings cannot be shared at this time as the data also forms part of an ongoing study.

Code availability Not applicable

Authors' contributions Conceptualization: Bei Li; Methodology: Lei Pan, Changan Liu, Xu Zhang, Bei Li; Formal analysis and investigation: Lei Pan, Changan Liu; Writing - original draft preparation: Bei Li; Writing - review \& editing: Bei Li; Supervision: Bei Li

\section{References}

1. Balasubramanian B, Das B, Skomski R, Zhang WY, Sellmyer DJ (2013) Novel nanostructured rareearth-free magnetic materials with high energy products. Adv Mater 25:6090-6093. https://doi.org/10.1002/adma.201302704

2. Gong X, Jiang YR, Zhang CC, Yang L, Li M, Ma H (2016) Investigation of the magnetic nickel nanoparticle on performance improvement of P3HT:PCBM solar cell. Appl Phys A 122:287. https://doi.org/10.1007/s00339-016-9809-0

3. Yu P, Zhang FL, Li ZY, Zhong ZQ, Govorov A, Fu L, Tan H, Jagadish C, Wang ZM (2018) Giant optical pathlength enhancement in plasmonic thin film solar cells using core-shell nanoparticles. J Phys D: Appl Phys 51:295106. https://doi.org/10.1088/1361-6463/aacb9d

4. Prins F, Monrabal-Capilla M, Osorio EA, Coronado E, van der Zant HSJ (2011) Room-temperature electrical addressing of a bistable spin-crossover molecular system. Adv Mater 23:1545-1549. https://doi.org/10.1002/adma.201003821

5. Usmani S, Mikolasek M, Gillet A, Sanchez Costa J, Rigoulet M, Chaudret B, Bousseksou A, LassalleKaiser B, Demont P, Molnár G, Salmon L, Carrey J, Tricard S (2020) Spin crossover in Fe(triazole)-Pt 
nanoparticle self-assembly structured at the sub-5 nm scale. Nanoscale 12:8180-8187. https://doi.org/10.1039/D0NR02154G

6. Bobo JF, Gabillet L, Bibes M (2004) Recent advances in nanomagnetism and spin electronics. J Phys Condens Matter 16:S471-S496. https://doi.org/10.1088/0953-8984/16/5/008

7. Yan JM, Zhang XB, Han S, Shioyama H, Xu Q (2008) Iron-nanoparticle-catalyzed hydrolytic dehydrogenation of ammonia borane for chemical hydrogen storage. Angew Chem Int Ed 47:22872289. https://doi.org/10.1002/anie.200704943

8. Meffre A, Mehdaoui B, Connord V, Carrey J, Fazzini PF, Lachaize S, Respaud M, Chaudret B (2015) Complex nano-objects displaying both magnetic and catalytic properties: a proof of concept for magnetically induced heterogeneous catalysis. Nano Lett 15:3241-3248.

https://doi.org/10.1021/acs.nanolett.5b00446

9. Yang SL, Peng L, Cao CY, Wei F, Liu J, Zhu YN, Liu C, Wang XS, Song WG (2016) Preparation of magnetic tubular nanoreactors for highly efficient catalysis. Chem: Asian J 11:2797-2801. https://doi.org/10.1002/asia.201600454

10. Pankhurst QA, Connolly J, Jones SK, Dobson J (2003) Applications of magnetic nanoparticles in biomedicine. J Phys D: Appl Phys 36:R167-R181. https://doi.org/10.1088/0022-3727/36/13/201

11. Koh I, Josephson L (2009) Magnetic nanoparticle sensors. Sensors 9:8130-8145. https://doi.org/10.3390/s91008130

12. Mohammad R, Kandasamy R (2017) Nanoparticle shapes on electric and magnetic force in water, ethylene glycol and engine oil based Cu, Al203 and SWCNTs. J Mol Liq 237:54-64. https://doi.org/10.1016/j.molliq.2017.04.045

13. Ozel F, Kockar H (2015) Growth and characterizations of magnetic nanoparticles under hydrothermal conditions: Reaction time and temperature. J Magn Magn Mater 373:213-216.

https://doi.org/10.1016/j.jmmm.2014.02.072

14. Sun M, Zangari G, Shamsuzzoha M, Metzger RM (2001) Electrodeposition of highly uniform magnetic nanoparticle arrays in ordered alumite. Appl Phys Lett 78:2964-2966.

https://doi.org/10.1063/1.1370986

15. Budi S, Daud AR, Radiman S, Umar AA (2010) Effective electrodeposition of Co-Ni-Cu alloys nanoparticles in the presence of alkyl polyglucoside surfactant. Appl Surf Sci 257:1027-1033. https://doi.org/10.1016/j.apsusc.2010.07.103

16. Seto T, Koga K, Akinaga H, Takano F, Orii T, Hirasawa M (2006) Laser ablation synthesis of monodispersed magnetic alloy nanoparticles. J Nanoparticle Res 8:371-378. https://doi.org/10.1007/s11051-005-9010-4

17. Lasemi N, Pacher U, Zhigilei LV, Bomati-Miguela O, Lahoz R, Kautek W (2018) Pulsed laser ablation and incubation of nickel, iron and tungsten in liquids and air. Appl Surf Sci 433:772-779. https://doi.org/10.1016/j.apsusc.2017.10.082

18. Simchi A, Ahmadi R, Reihani SMS, Mahdavi A (2007) Kinetics and mechanisms of nanoparticle formation and growth in vapor phase condensation process. Mater Des 28:850-856. 
https://doi.org/10.1016/j.matdes.2005.10.017

19. Grammatikopoulos P, Steinhauer S, Vernieres J, Singh V, Sowwan M (2016) Nanoparticle design by gas-phase synthesis. Adv Phys: X 1:81-100. https://doi.org/10.1080/23746149.2016.1142829

20. Shyjumon I, Gopinadhan M, Ivanova O, Quaas M, Wulff H, Helm CA, Hippler R (2006) Structural deformation, melting point and lattice parameter studies of size selected silver clusters. Eur Phys J D 37:409-415. https://doi.org/10.1140/epjd/e2005-00319-x

21. Li ZY, Young NP, Di Vece M, Palomba S, Palmer RE, Bleloch AL, Curley BC, Johnston RL, Jiang J, Yuan $\mathrm{J}(2008)$ Three-dimensional atomic-scale structure of size-selected gold nanoclusters. Nature 451:46-48. https://doi.org/10.1038/nature06470

22. Singh V, Cassidy C, Grammatikopoulos P, Djurabekova F, Nordlund K, Sowwan M (2014) Heterogeneous gas-phase synthesis and molecular dynamics modeling of Janus and core-satellite Si-Ag nanoparticles. J Phys Chem C 118:13869-13875. https://doi.org/10.1021/jp500684y

23. Bohra M, Grammatikopoulos P, Singh V, Zhao J, Toulkeridou E, Steinhauer S, Kioseoglou J, Bobo J-F, Nordlund K, Djurabekova F, Sowwan M (2017) Tuning the onset of ferromagnetism in heterogeneous bimetallic nanoparticles by gas phase doping. Phys Rev Mater 1:066001. https://doi.org/10.1103/PhysRevMaterials.1.066001

24. Golkar F, Kramer MJ, Zhang Y, McCallum RW, Skomski R, Sellmyer DJ, Shield JE (2012) Structure and magnetic properties of Co-W clusters produced by inert gas condensation. J Appl Phys 111:07B524. https://doi.org/10.1063/1.3676425

25. Lümmen N, Kraska T (2006) Influence of the carrier gas on the formation of iron nano-particles from the gas phase: A molecular dynamics simulation study. Comput Mater Sci 35:210-215. https://doi.org/https://doi.org/10.1016/j.commatsci.2004.07.008

26. Kesälä E, Kuronen A, Nordlund K (2007) Molecular dynamics simulation of pressure dependence of cluster growth in inert gas condensation. Phys Rev B 75:174121. https://doi.org/10.1103/PhysRevB.75.174121

27. Akbarzadeh H, Abbaspour M, Masoumi A, Mehrjouei E (2017) Dynamical investigation of formation of NiPt nanoclusters in gas phase. J Mol Liq 240:221-224. https://doi.org/https://doi.org/10.1016/j.molliq.2017.05.109

28. Akbarzadeh H, Abbaspour M, Mehrjouei E, Masoumi A (2017) Structural evolution of Pt/Pd nanoparticles in condensation process. J Mol Liq 248:822-829.

https://doi.org/https://doi.org/10.1016/j.molliq.2017.10.132

29. Abbaspour M, Valizadeh Z, Jorabchi MN (2019) Nucleation, coalescence, thermal evolution, and statistical probability of formation of $\mathrm{Au} / \mathrm{Ir} / \mathrm{Pd}$ nanoalloys in gas-phase condensation process. $\mathrm{J} \mathrm{Mol}$ Liq 274:434-446. https://doi.org/https://doi.org/10.1016/j.molliq.2018.10.152

30. Zhao Y, Ni C, Kruczynski D, Zhang X, Xiao JQ (2004) Exchange-coupled soft magnetic FeNi - SiO2 nanocomposite. J Phys Chem B 108:3691-3693. https://doi.org/10.1021/jp037588r

31. Xu MH, Zhong W, Qi XS, Au CT, Deng Y, Du YW (2010) Highly stable Fe-Ni alloy nanoparticles encapsulated in carbon nanotubes: Synthesis, structure and magnetic properties. J Alloys Compd 
495:200-204. https://doi.org/https://doi.org/10.1016/j.jallcom.2010.01.121

32. Ibrahim EMM, Hampel S, Wolter AUB, Kath M, El-Gendy AA, Klingeler R, Taschner C, Khavrus VO, Gemming T, Leonhardt A, Buchner B (2012) Superparamagnetic FeCo and FeNi Nanocomposites Dispersed in Submicrometer-Sized C Spheres. J Phys Chem C 116:22509-22517. https://doi.org/10.1021/jp304236x

33. Bonny G, Castin N, Terentyev D (2013) Interatomic potential for studying ageing under irradiation in stainless steels: the FeNiCr model alloy. Modell Simul Mater Sci Eng 21:085004. https://doi.org/

34. Pal S, Meraj M (2016) Structural evaluation and deformation features of interface of joint between nano-crystalline $\mathrm{Fe}-\mathrm{Ni}-\mathrm{Cr}$ alloy and nano-crystalline Ni during creep process. Mater Des 108:168182. https://doi.org/10.1016/j.matdes.2016.06.086

35. Sak-Saracino E, Urbassek HM (2016) The $\mathrm{a} \leftrightarrow \mathrm{y}$ transformation of an Fe1-xCrx alloy: a moleculardynamics approach. Int J Mod Phys C 27:1650124. https://doi.org/10.1142/S0129183116501242

36. Wu C, Lee BJ, Su X (2017) Modified embedded-atom interatomic potential for Fe-Ni, Cr-Ni and Fe-CrNi systems. Calphad 57:98-106. https://doi.org/10.1016/j.calphad.2017.03.007

37. Zhang X, Li B, Liu HX, Zhao GH, Yang QL, Cheng XM, Wong CH, Zhang YM, Lim CWJ (2019) Atomic simulation of melting and surface segregation of ternary Fe-Ni-Cr nanoparticles. Appl Surf Sci 465:871-879. https://doi.org/10.1016/j.apsusc.2018.09.257

38. Li B, Zheng R, Zhang X, Zhao GH, Liu HX (2020) Molecular dynamics simulation of inert gas condensation of ternary Fe-Ni-Cr nanoparticles. Comput Mater Sci 177:109579. https://doi.org/10.1016/j.commatsci.2020.109579

39. Ziegler JF, Biersack JP, Littmark U, Pergamon, Boston, MA, USA 1985

40. Akbarzadeh H, Shamkhali AN, Mehrjouei E (2017) Ag-Au bimetallic nanoclusters formed from a homogeneous gas phase: a new thermodynamic expression confirmed by molecular dynamics simulation. Phys Chem Chem Phys 19:3763-3769. https://doi.org/10.1039/C6CP08508C

41. Wu YQ, Shen T, Lu XG (2013) Evolutions of lamellar structure during melting and solidification of Fe9577 nanoparticle from molecular dynamics simulations. Chem Phys Lett 564:41-46. https://doi.org/10.1016/j.cplett.2013.01.070

42. Hou M (2017) Solid-liquid and liquid-solid transitions in metal nanoparticles. Phys Chem Chem Phys 19:5994-6005. https://doi.org/10.1039/c6cp08606c

43. Shibuta Y, Suzuki T (2010) Melting and solidification point of fcc-metal nanoparticles with respect to particle size: A molecular dynamics study. Chem Phys Lett 498:323-327. https://doi.org/10.1016/j.cplett.2010.08.082

44. Shen T, Meng W, Wu Y, Lu X (2013) Size dependence and phase transition during melting of fcc-Fe nanoparticles: A molecular dynamics simulation. Appl Surf Sci 277:7-14. https://doi.org/10.1016/j.apsusc.2013.03.017

\section{Figures}




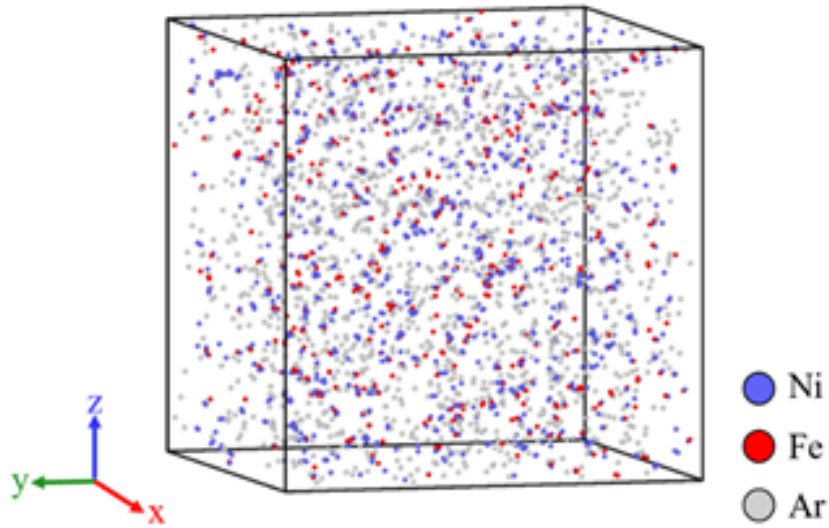

\section{Figure 1}

Atomic configuration of a typical equilibrated inert-gas-metal-vapor mixture consisting of $1500 \mathrm{Ar}$ atoms and 1500 metal atoms with $30 \mathrm{wt} . \% \mathrm{Fe}$ at $\mathrm{T}=4000 \mathrm{~K}$.

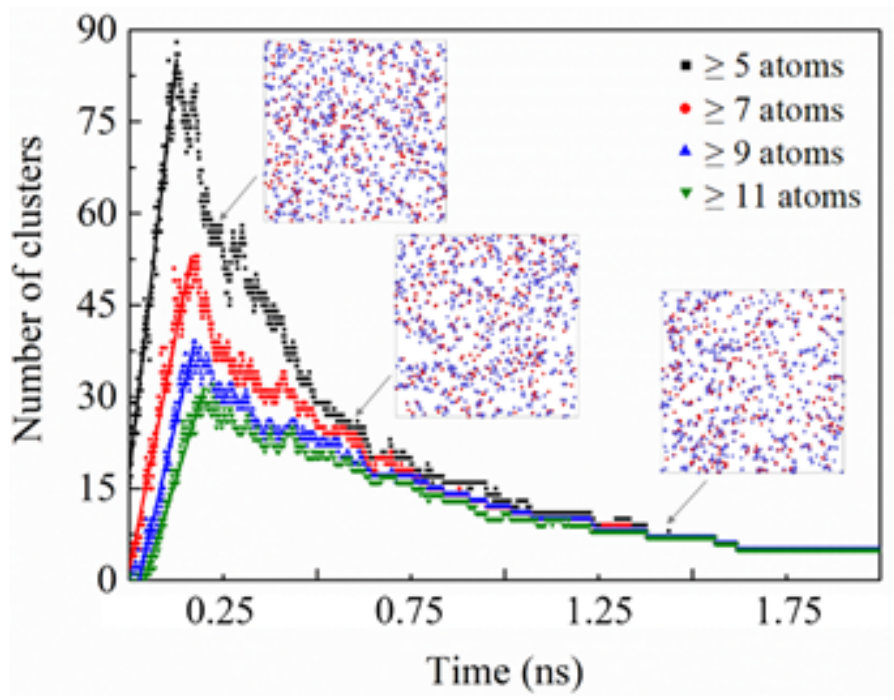

\section{Figure 2}

Number of the created $\mathrm{Ni}$-Fe clusters as a function of simulation time with different thresholds $(5,7,9$ and 11 atoms) for the model with $30 \mathrm{wt} . \% \mathrm{Fe}$ and $\mathrm{Ar} / \mathrm{Metal}=1: 1$. The insets are the snapshots of the formation of small Ni-Fe clusters via surface growth by consuming basal metal atoms, i.e., monomers, dimers and trimers. The solid lines are ascending linear fitted curves, and Fe and Ni atoms are colored red and purple. 


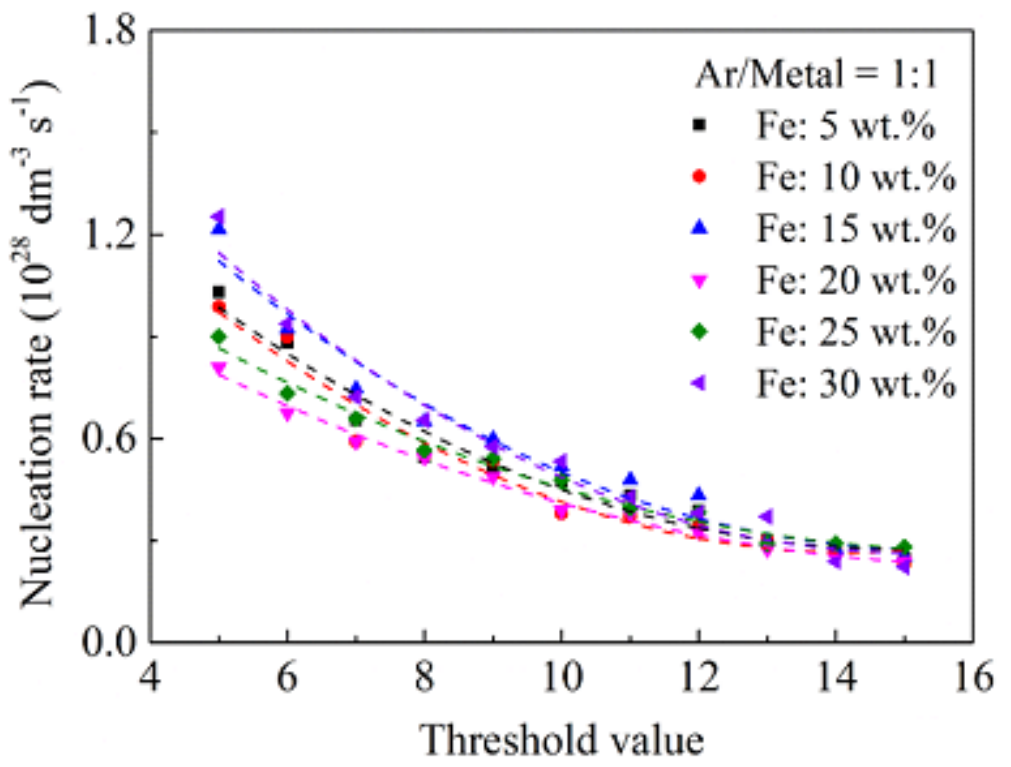

(a)

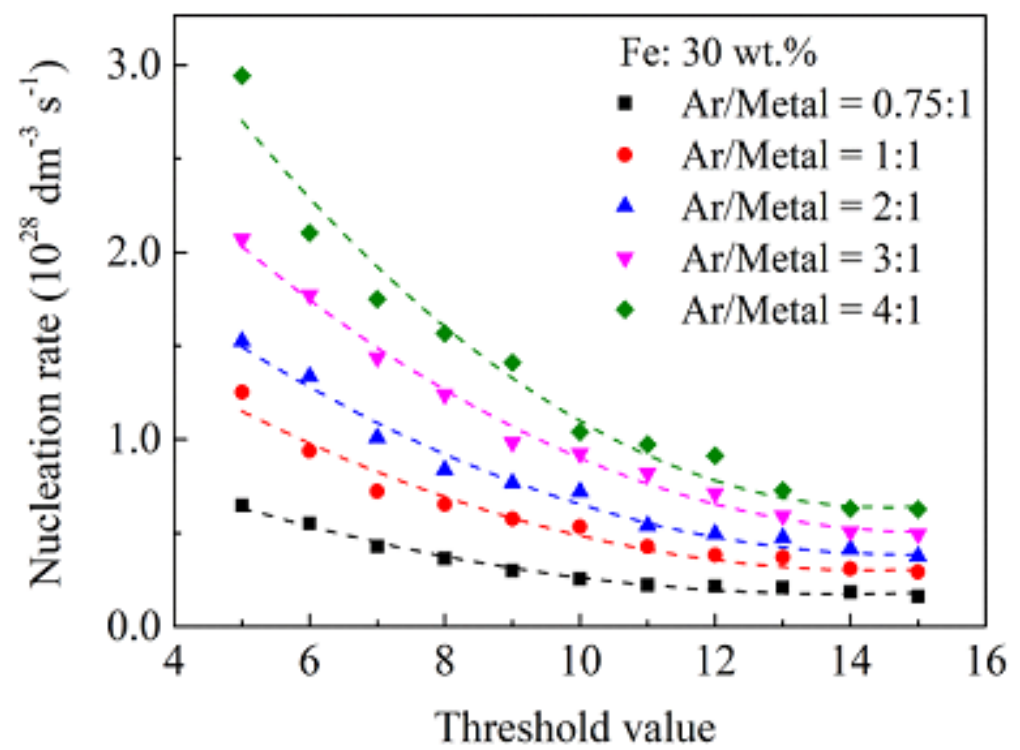

(b)

\section{Figure 3}

Nucleation rates determined from the slope of the fitted curves in Fig. 2 divided by the simulation cell volume for different (a) Fe concentrations and (b) amount of inert gas. The dash lines are the exponential fitted curves. 


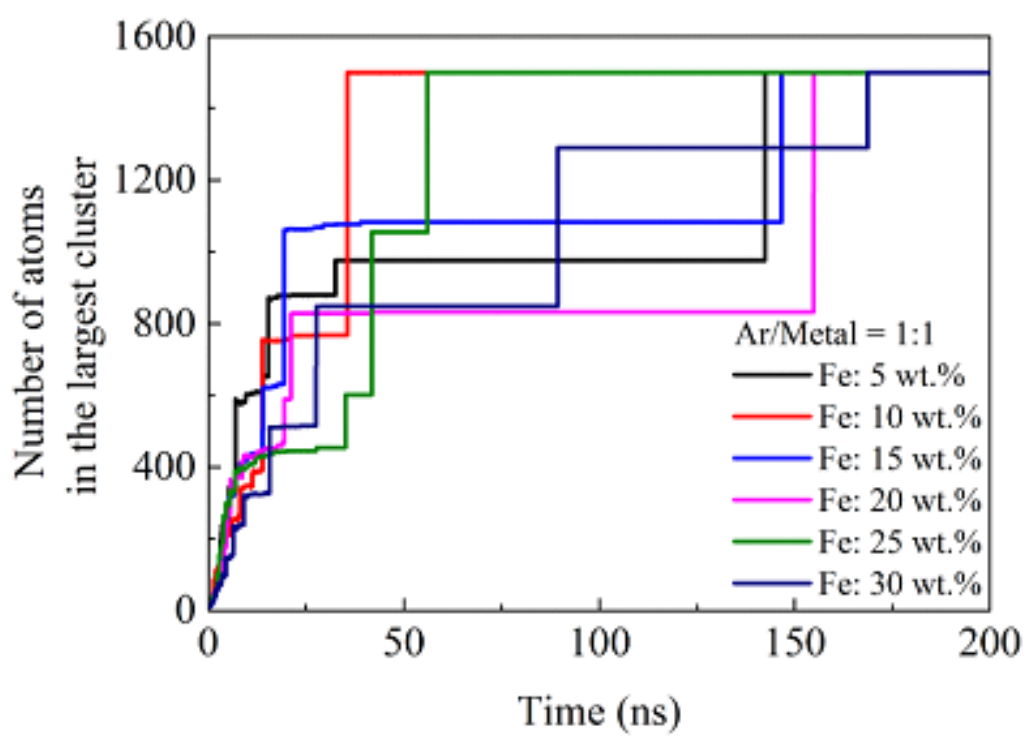

(a)

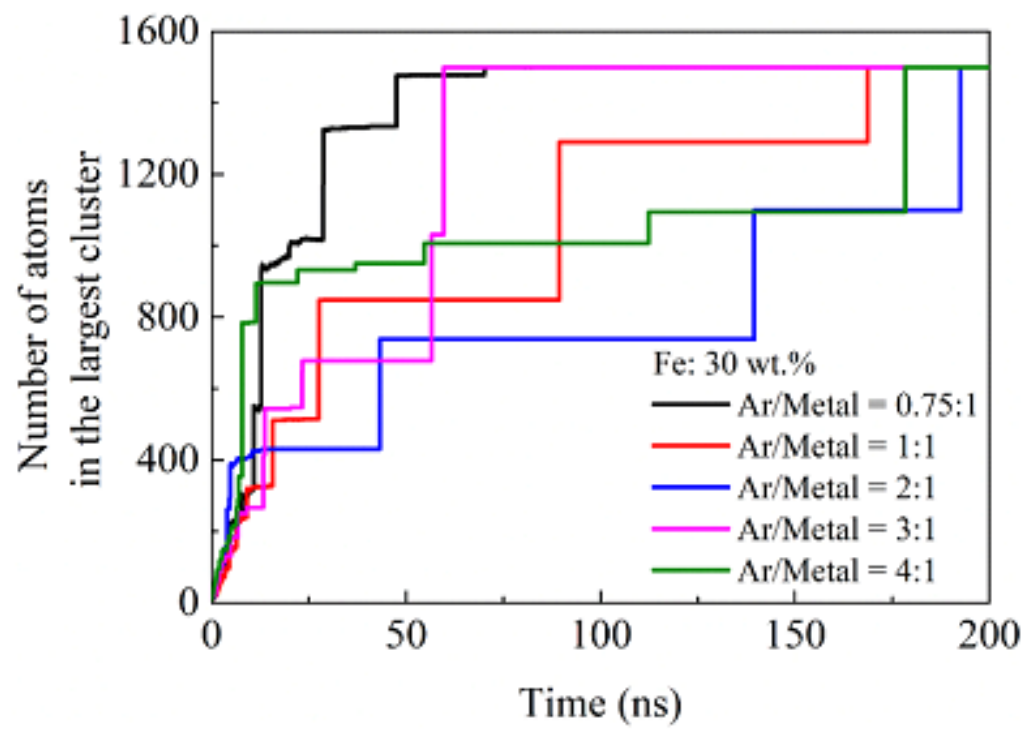

(b)

\section{Figure 4}

Number of atoms in the largest cluster as a function of simulation time for different (a) Fe concentrations and (b) amount of inert gas. 
(a)

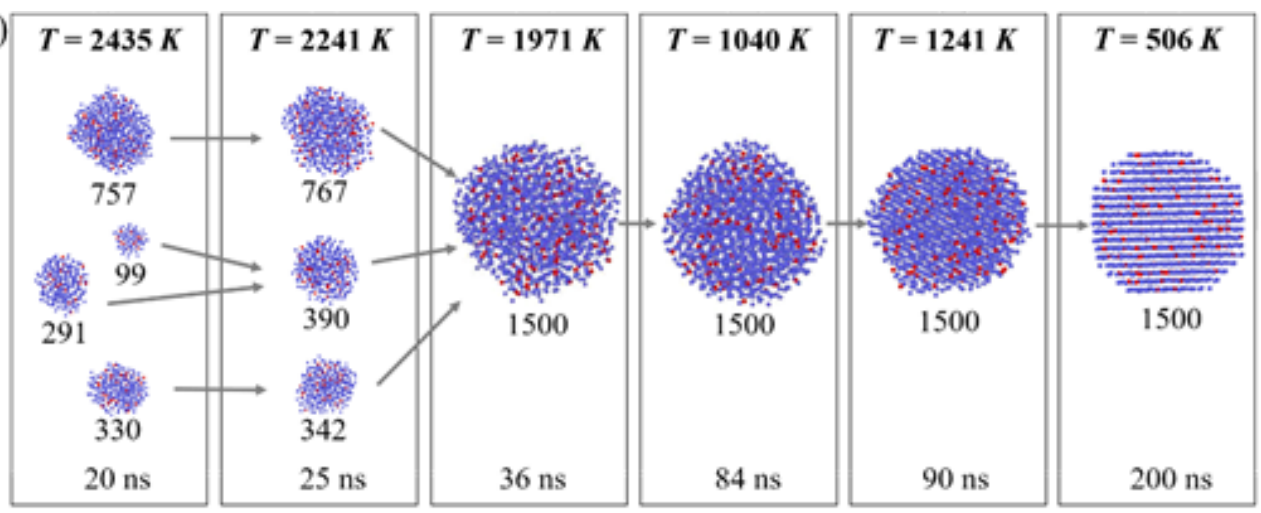

(b)

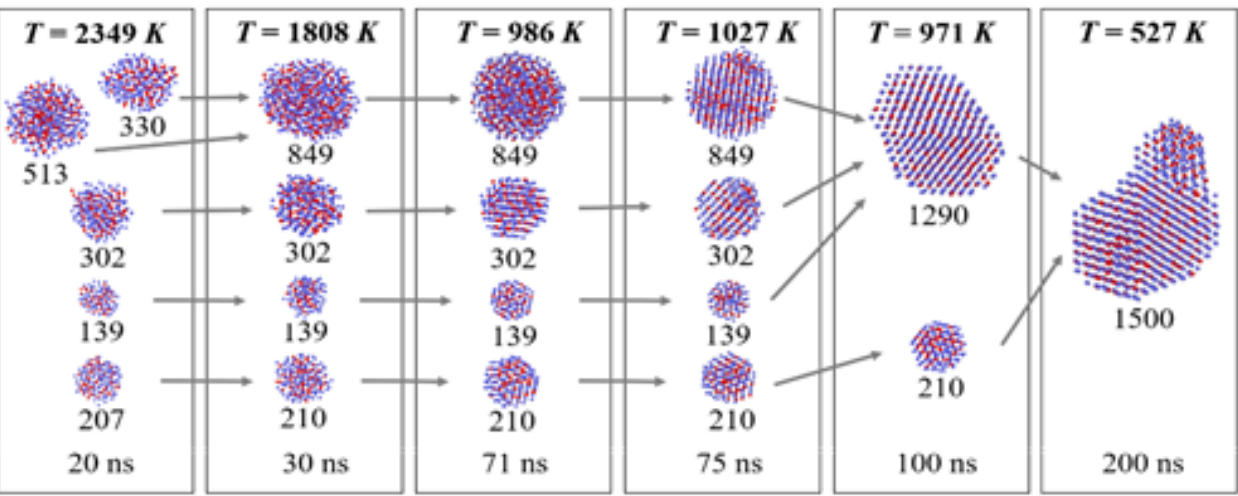

(c)
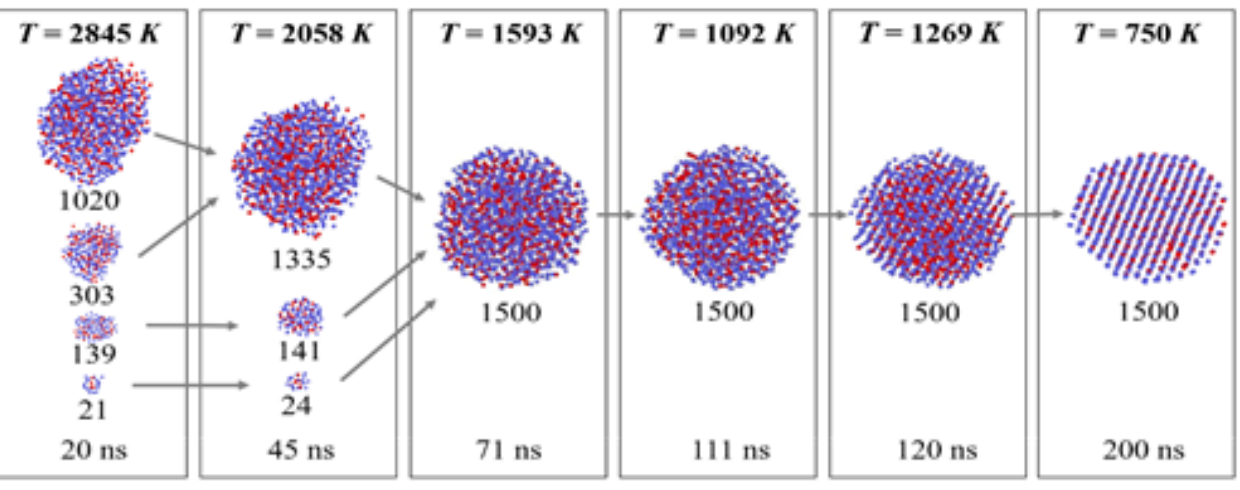

(d)

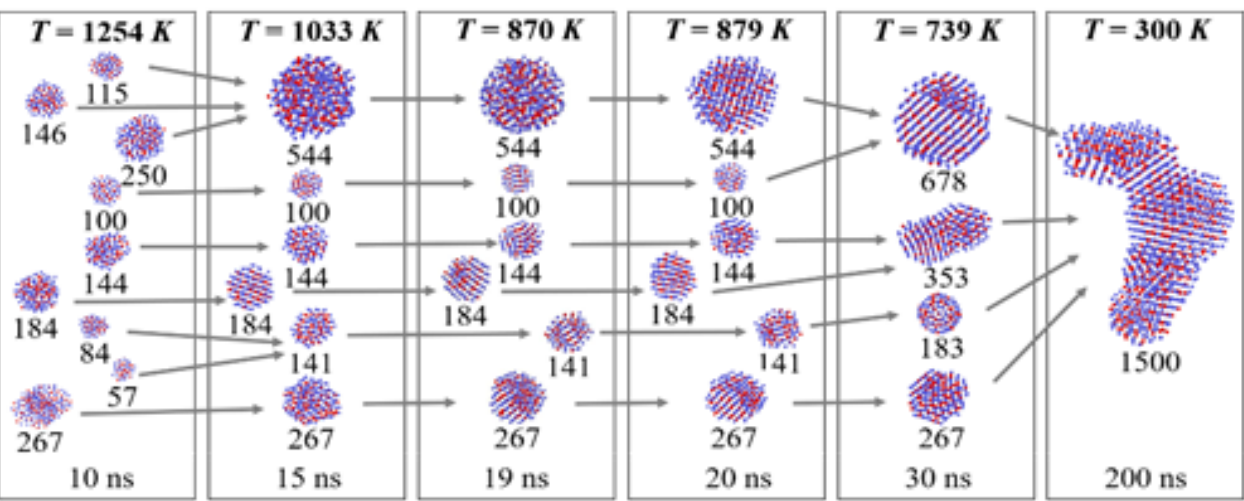

Figure 5

Snapshots of the growth of Ni-Fe nanoparticles for (a) Fe: 10 wt.\%, Ar/Metal = 1:1, (b) Fe: 30 wt.\%, Ar/Metal = 1:1, (c) Fe: 30 wt.\%, Ar/Metal = 0.75:1 and (d) Fe: 30 wt.\%, Ar/Metal = 3:1 at different metal temperatures $\mathrm{T}$ and simulation time t. The number indicates the amount of metal atoms for each cluster. Fe and $\mathrm{Ni}$ atoms are colored red and purple. 


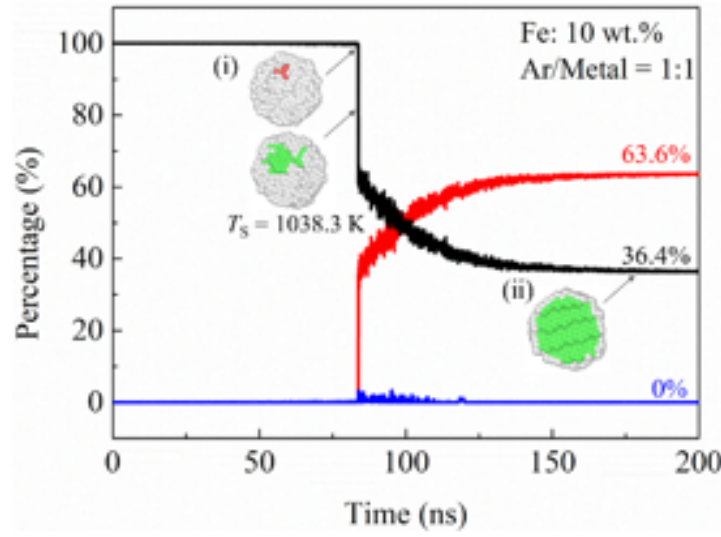

(a)

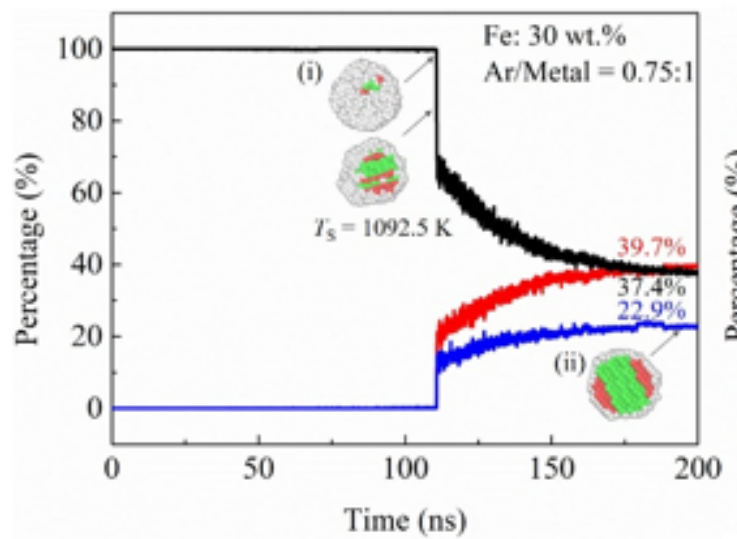

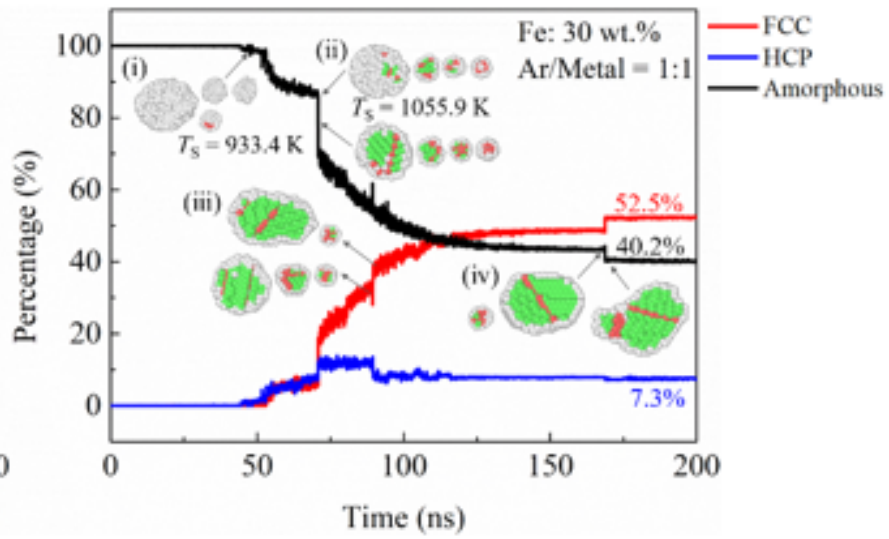

(b)

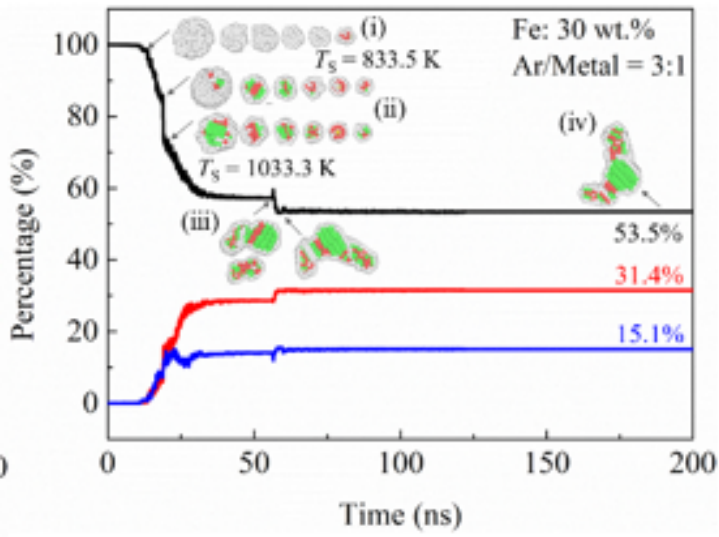

Figure 6

Percentage evolution of FCC, $\mathrm{HCP}$ and other amorphous structure as a function of simulation time for $\mathrm{Ni}$ Fe nanoparticles: (a) Fe: 10 wt.\%, Ar/Metal = 1:1, (b) Fe: 30 wt.\%, Ar/Metal = 1:1, (c) Fe: 30 wt.\%, Ar/Metal $=0.75: 1$ and (d) Fe: $30 \mathrm{wt} . \%, \mathrm{Ar} / \mathrm{Metal}=3: 1$. The insets are the CNA images of the clusters at specific times which denote the solidification (i.e., $i$ in (a) and (c), $i$ and ii in (b) and (d)), cluster aggregation (iii in (b) and (d) ) and structural relaxation (ii in (a) and (c), iv in (d)). The FCC, HCP and amorphous structure in the CNA images are colored as green, red and light gray, respectively.

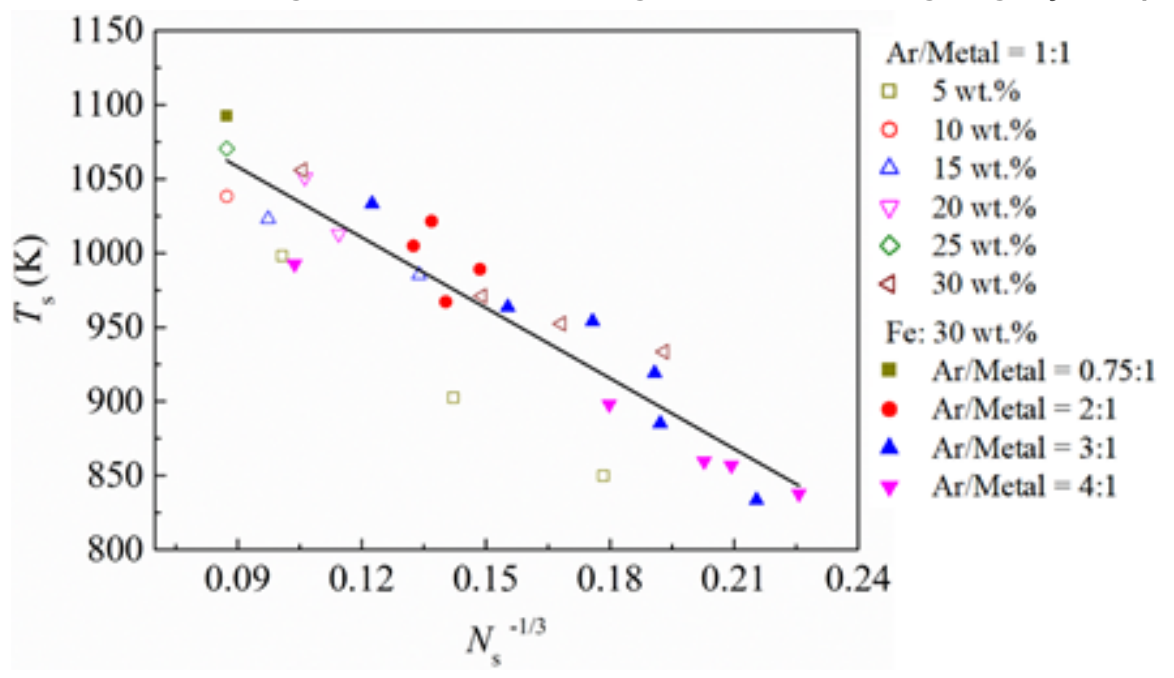


Figure 7

Solidification temperature Ts as a function of the reciprocal of Ns $1 / 3$ for the Ni-Fe cluster $\mathrm{S}$ with different Fe concentrations and amount of inert gas. The solid line is the linear fitted curve for the MD simulation results.

\section{Supplementary Files}

This is a list of supplementary files associated with this preprint. Click to download.

- GraphicalAbstract.docx

- AppendixA.Supplementarymaterial.docx 